S. Patel - J. A. L. Minton - M. N. Weedon •

T. M. Frayling • C. Ricketts · G. A. Hitman •

M. I. McCarthy • A. T. Hattersley • M. Walker •

T. G. Barrett

\title{
Common variations in the ALMS1 gene do not contribute to susceptibility to type 2 diabetes in a large white UK population
}

Received: 14 December 2005 / Accepted: 2 February 2006 / Published online: 7 April 2006

C) Springer-Verlag 2006

\begin{abstract}
Aims/hypothesis: Alström syndrome is a rare monogenic disorder characterised by retinal dystrophy, deafness and obesity. Patients also have insulin resistance, central obesity and dyslipidaemia, thus showing similarities with type 2 diabetes. Rare mutations in the ALMS1 gene cause severe gene disruption in Alström patients; however, ALMS1 gene polymorphisms are common in the general population. The aim of our study was to determine whether common variants in $A L M S 1$ contribute to susceptibility to type 2 diabetes in the UK population. Methods: Direct sequencing was performed on coding
\end{abstract}

Electronic Supplementary Material Supplementary material is available for this article at http://10.1007/s00125-006-0227-2

S. Patel $\cdot$ M. Walker

School of Clinical Medical Sciences,

University of Newcastle,

Newcastle upon Tyne, UK

J. A. L. Minton - C. Ricketts - T. G. Barrett Department of Medical and Molecular Genetics, The Medical School, University of Birmingham, Birmingham, UK

M. N. Weedon - T. M. Frayling · A. T. Hattersley

Institute of Biomedical and Clinical Science,

Peninsula Medical School,

Exeter, UK

G. A. Hitman

Centre for Diabetes and Metabolic Medicine,

Queen Mary's School of Medicine and Dentistry,

London, UK

M. I. McCarthy

Department of Endocrinology and Metabolism,

Oxford Centre for Diabetes,

Oxford, UK

T. G. Barrett $(\bowtie)$

Diabetes Unit, Birmingham Children's Hospital,

Steelhouse Lane,

Birmingham, B4 6NH, UK

e-mail: T.G.Barrett@bham.ac.uk

Tel.: +44-121333-9267

Fax: +44-121333-9272 regions and intron/exon boundaries of the ALMS1 gene in 30 unrelated probands with type 2 diabetes. The linkage disequilibrium (LD; $D^{\prime}$ and $r^{2}$ ) and haplotype structure were examined for the identified variants. The common (minor allele frequency $[\mathrm{MAF}]>5 \%$ ) single-nucleotide polymorphisms tagging the common haplotypes (tagged SNPs [tSNPs]) were identified and genotyped in 1985 subjects with type 2 diabetes, 2,047 control subjects and 521 families. Results: We identified 18 variants with MAF between 6 and 38\%. Three SNPs efficiently tagged three common haplotypes (rs1881245, rs3820700 and rs1320374). There was no association (all $p>0.05$ ) between the tSNPs and type 2 diabetes in the case-control study and minor alleles of the tSNPs were not overtransmitted to probands with type 2 diabetes in the family study. Conclusions/interpretation: Common variations in the ALMS1 gene were not associated with type 2 diabetes in a large study of a white UK population.

Keywords ALMS1 - Alström syndrome · Common variation · Haplotypes $\cdot$ Type 2 diabetes

Abbreviations ESM: Electronic Supplementary Material $\cdot$ LD: linkage disequilibrium - MAF: minor allele frequency - SNP: single-nucleotide polymorphism - TDT: transmission disequilibrium test - tSNP: tagged singlenucleotide polymorphism

\section{Introduction}

The study of monogenic diabetes has led to the identification of a number of type 2 diabetes susceptibility genes. For instance, common variation in the HNF4A gene can predispose to type 2 diabetes in the general population [1]. Alström syndrome (OMIM 203800) [2] is a recessive form of monogenic diabetes characterised by retinal dystrophy, sensorineural deafness, cardiomyopathy and childhoodonset obesity, leading to type 2 diabetes (in $80 \%$ of Alström syndrome patients by the age of 16 years), insulin resistance, central adiposity and hypertriglyceridaemia 
[3]. The phenotype suggests that mutations in the Alström gene lead to both obesity and diabetes, and obligate heterozygotes may be at increased risk of diabetes [2].

The ALMS1 gene maps to chromosome 2p13, a region linked to type 2 diabetes [4]. It comprises 23 exons spanning over $224 \mathrm{~kb}$ of genomic DNA encoding a protein of 4,169 amino acids of unknown function [5]. Rare mutations in ALMS1 segregate with Alström syndrome in affected families $[5,6]$.

We aimed to determine whether common variants of ALMS1 are associated with type 2 diabetes in white individuals in the UK. We identified 18 common ALMS1 variants and three common haplotypes in 30 subjects with type 2 diabetes, and then used case-control and familybased methods to test for association between the variants and type 2 diabetes.

\section{Subjects and methods}

\section{Subjects}

The populations used for the analysis and the inclusion and exclusion criteria have been described elsewhere [7]. Briefly, all case subjects were unrelated white UK citizens with type 2 diabetes, recruited from three sources: probands from type 2 diabetes sibships from the Diabetes UK Warren 2 repository $(n=559)$, a new collection of individuals with type 2 diabetes from the Warren 2 repository $(n=1,141)$ and a collection of subjects with young-onset type 2 diabetes (aged $>18$ and $<45$ years at diagnosis of type 2 diabetes) $(n=285)$. The control subjects were white UK citizens recruited from two sources: parents from a consecutive birth cohort (the Exeter Family Study) $(n=1,574)$ and a nationally recruited population-based control sample of blood donors without known diabetes from the European Collection of Cell Cultures $(n=473)$. The families consisted of an affected proband with type 2 diabetes and both parents ( $n=402)$ (Warren 2 trios) or one parent and at least one unaffected sibling $(n=119)$ (Warren 2 duos) [8]. The family study individuals were independent of the case-control study. The study was approved by local ethics committees and written consent was obtained for all subjects.

\section{Sequencing}

Common variation in the ALMS1 gene was identified by direct sequencing of coding regions and exon/intron boundaries using 30 randomly selected unrelated patients with type 2 diabetes from the Warren parent-offspring trio collection [8]. Coding regions and exon/intron boundaries were divided into overlapping fragments and amplified by PCR (primers and conditions are available from the authors). The fragments were sequenced in both directions using the BigDye Terminator chemistry method either on an ABI 3730 sequencer (Applied Biosystems, Warrington, UK) or a CEQ 8000 genetic analyser (Beckman Coulter, Fullerton, CA, USA).

\section{Genotyping}

Genotyping was carried out by Kbiosciences (Hoddesdon, UK) using the TaqMan system (Applied Biosystems). Of the genotyped samples, $10 \%$ were duplicates and there was at least one negative control per 96-well plate. Genotyping accuracy was determined by the genotype concordance between duplicate samples and was greater than $99.6 \%$ for each of the tagged single-nucleotide polymorphisms (tSNPs). The genotyping success rate for each tSNP was as follows: case subjects, rs $1881245,96 \%$, rs 3820700 , 98\%, rs1320374, 95\%; control subjects, rs $1881245,97 \%$, rs3820700, 97\%, rs $1320374,96 \%$. There were no Mendelian inheritance errors in the families. All case, control

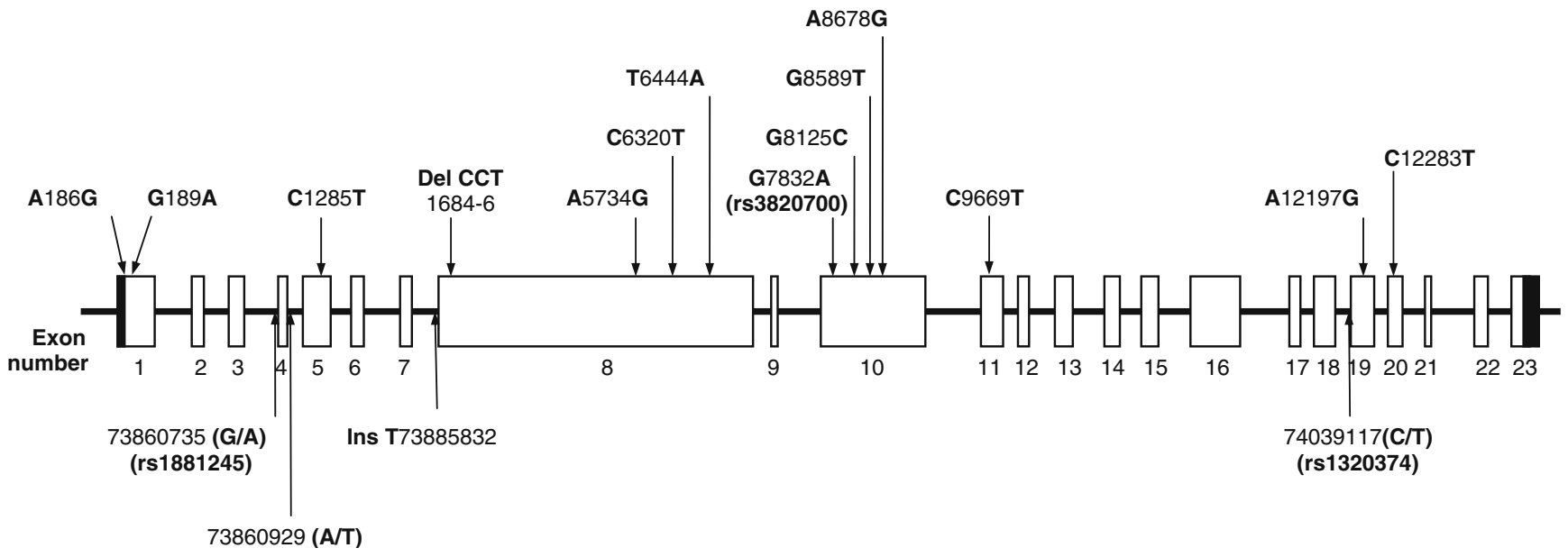

Fig. 1 Schematic representation of the ALMS1 gene. Exons are shown in white boxes and the $3^{\prime}$ and $5^{\prime}$ untranslated regions in black boxes. The locations of variants identified by sequencing 30 unrelated type 2 diabetes probands are shown. Intronic variants are numbered according to chromosome 2 nucleotide position using genomic contig NT_022184 http://www.ncbi.nlm.nih.gov, last accessed in February 2006). The tagged SNPs are shown with their dbSNP rs identification numbers. Ins, insertion; Del, deletion 
Table 1 Clinical characteristics of the study subjects

\begin{tabular}{|c|c|c|c|c|c|c|c|}
\hline & \multicolumn{3}{|l|}{ Cases } & \multicolumn{2}{|l|}{ Controls } & \multicolumn{2}{|l|}{ Families } \\
\hline & $\begin{array}{l}\text { Warren } 2 \\
\text { probands }\end{array}$ & $\begin{array}{l}\text { Warren } 2 \\
\text { cases }\end{array}$ & $\begin{array}{l}\text { Young-onset type } 2 \\
\text { diabetes }\end{array}$ & $\begin{array}{l}\text { Exeter Family } \\
\text { Study }\end{array}$ & ECACC & $\begin{array}{l}\text { Warren } 2 \text { family } \\
\text { trios }^{\mathrm{d}}\end{array}$ & Warren 2 duos $^{\mathrm{d}}$ \\
\hline Number $(n)$ & 559 & 1,141 & 285 & 1,574 & 473 & 402 & 119 \\
\hline Male $(\%)$ & 54 & 61 & 55 & 49 & $52^{\mathrm{c}}$ & 58 & 58 \\
\hline $\begin{array}{l}\text { Age at diagnosis }{ }^{\mathrm{a}} \\
\text { (years) }\end{array}$ & $56(50-61)$ & $52(45-57)$ & $40(35-44)$ & NA & NA & $40(35-45)$ & $45(39.5-49)$ \\
\hline BMI $\left(\mathrm{kg} / \mathrm{m}^{2}\right)$ & $28.1(25.3-31.5)$ & $30.7(27.3-35.1)$ & $32.2(27.8-36.6)$ & $26.2(24.0-28.9)^{\mathrm{b}}$ & NA & $32.2(28.4-37.5)$ & $32.2(28.2-36.2)$ \\
\hline $\begin{array}{l}\text { Treatment } \\
\text { (diet/OHA/ } \\
\text { insulin) (\%) }\end{array}$ & $18 / 67 / 15$ & $8 / 64 / 27$ & $9 / 53 / 38$ & NA & NA & $21 / 64 / 16$ & $15 / 57 / 28$ \\
\hline
\end{tabular}

Only subjects with genotype data for at least one SNP are included

Data are median (interquartile range)

Clinical details were not available for ECACC population control samples

Control study populations were not on treatment for diabetes

ECACC Population-based control sample of blood donors from the European Collection of Cell Cultures, $O H A$ oral hypoglycaemic agents

${ }^{\mathrm{a}} \mathrm{Age}$ at diagnosis for case subjects, age at time of study for control subjects

${ }^{\mathrm{b}} \mathrm{BMI}$ measurement for men only because women were pregnant at the time of the study

${ }^{c}$ Percentage of males was determined by XY PCR

${ }^{\mathrm{d}}$ Only probands

and family cohorts were in Hardy-Weinberg equilibrium ( $\chi^{2}$ test, $p>0.05$ ), except for the Warren 2 case subjects for SNP rs 3820700 ( $\chi^{2}$ test, $\left.p=0.04\right)$. Given the other quality control results and the similarity of linkage disequilibrium (LD) between the SNPs in all our cohorts (and the HapMapII data, where the $D^{\prime}$ between SNPs is 1), we suggest that the mild deviation from Hardy-Weinberg equilibrium was due to chance variation and multiple testing rather than genotyping error.

\section{Statistical analysis}

We performed analysis of the $\operatorname{LD}\left(D^{\prime}\right.$ and $\left.r^{2}\right)$ and haplotype structure of the ALMS1 gene using the Haploview program http://www.broad.mit.edu/mpg/haploview/index.php, version 3.2, last accessed in February 2006). For the casecontrol analysis, odds ratios with $95 \%$ CIs and $p$ values were determined using $\chi^{2}$ tests. For the family data we used the transmission disequilibrium test (TDT)/sibTDT of Spielman and Ewens [9]. Family trios were excluded from the analysis if the genotype data for parents were missing. The trios were also analysed using the TRANSMIT program http://www-gene.cimr.cam.ac.uk/clayton/ software/transmit.txt), and the results were very similar to those obtained by the first method.

\section{Results}

We identified 18 variants across the ALMS1 gene with a minor allele frequency (MAF) between 6 and 38\% (Fig. 1). These included three intronic and 13 coding SNPs (four synonymous and nine non-synonymous). We also identified two novel insertion/deletion variants (not previously reported in the SNP databases [dbSNP], http://www.ncbi.

Table 2 Association of the ALMS1 tagged SNPs based on 1985 case subjects with type 2 diabetes and 2,047 control subjects

\begin{tabular}{|c|c|c|c|c|c|c|c|c|}
\hline \multirow{3}{*}{$\frac{\mathrm{tSNP}}{\mathrm{rs} 1881245}$} & \multirow{2}{*}{\multicolumn{3}{|c|}{ Genotype frequency $(n)$}} & \multirow{3}{*}{$p$ value } & \multicolumn{4}{|c|}{ Allele frequency $(n)$} \\
\hline & & & & & Allele 1 & Allele 2 & Odds ratio $(95 \% \mathrm{CI})$ & $p$ value \\
\hline & GG & GA & AA & & G & A & & \\
\hline Case & $58.2(1,112)$ & $36.2(691)$ & $5.6(107)$ & 0.76 & $76.3(2,915)$ & $23.7(905)$ & $1.03(0.93-1.14)$ & 0.57 \\
\hline Control & $57.7(1,144)$ & $36.2(717)$ & $6.1(122)$ & & $75.8(3,005)$ & $24.2(961)$ & & \\
\hline rs3820700 & GG & GA & AA & & $\mathrm{G}$ & A & & \\
\hline Case & $74.9(1,461)$ & $22.8(444)$ & $2.3(45)$ & 0.92 & $86.3(3,366)$ & $13.7(534)$ & $1.00(0.88-1.14)$ & 0.98 \\
\hline Control & $74.7(1,490)$ & $23.1(461)$ & $2.2(43)$ & & $86.3(3,441)$ & $13.7(547)$ & & \\
\hline rs1320374 & $\mathrm{CC}$ & $\mathrm{CT}$ & $\mathrm{TT}$ & & $\mathrm{C}$ & $\mathrm{T}$ & & \\
\hline Case & $39.4(745)$ & $47.1(889)$ & $13.5(255)$ & 0.70 & $63.0(2,379)$ & $37.0(1,399)$ & $1.01(0.96-1.16)$ & 0.81 \\
\hline Control & $39.7(780)$ & $46.0(903)$ & $14.3(281)$ & & $62.7(2,463)$ & $37.3(1,465)$ & & \\
\hline
\end{tabular}

$t$ SNP tagged SNP 
Table 3 TDT/sibTDT analysis of $A L M S 1$ tagged SNPs

\begin{tabular}{lllrl}
\hline tSNP & $\begin{array}{l}\text { Observed } \\
\text { transmission }\end{array}$ & $\begin{array}{l}\text { Expected } \\
\text { transmission }\end{array}$ & $\begin{array}{l}Z_{\max } \\
\text { score }^{\mathrm{a}}\end{array}$ & $\begin{array}{l}p \\
\text { value }^{\mathrm{b}}\end{array}$ \\
\hline rs1881245 & 179 & 179 & -0.04 & 0.9 \\
rs3820700 & 160 & 157 & 0.38 & 0.71 \\
rs1320374 & 228 & 233 & 0.48 & 0.63 \\
\hline
\end{tabular}

The TDT results are presented for the minor allele at each tSNP tSNP tagged SNP

${ }^{\mathrm{a}} Z_{\max }$ score from the TDT/sibTDT analysis

${ }^{\mathrm{b}}$ Two-tailed

nlm.nih.gov/SNP/, last accessed in February 2006), an inframe CCT deletion in exon 8 resulting in a proline amino acid deletion and a $\mathrm{T}$ insertion $64 \mathrm{bp}$ upstream of exon 8 . We found all of the validated common (MAF >5\%) dbSNPs in the regions we sequenced. Table 1 of the Electronic Supplementary Material (ESM) provides information on the variants.

We examined the LD structure for the identified variants using the Haploview program. In the subsample of 30 probands, three haplotypes that were tagged by three SNPs occurred at a frequency greater than $5 \%$ and accounted for $75 \%$ of all haplotypes. The three common haplotypes defined by the three tSNPs (rs1881245, rs3820700 and rs 1320374) were: G, G, C (54\%), G, A, T (13\%) and A, G, $\mathrm{T}(8 \%)$.

We used the HapMapII project CEPH (Utah residents with ancestry from northern and western Europe) trio data http://www.HapMap.org, last accessed in February 2006) to see how well our three tSNPs captured the common variation across the ALMS1 gene.

Overall, there was very good correlation between the SNPs across the ALMSI gene from the HapMapII data (ESM Fig. 1). We examined the extent of the LD using the Tagger program http://www.broad.mit.edu/mpg/tagger/, last accessed in February 2006); our three tSNPs captured almost all the common variation (MAF $>5 \%$ ) in the ALMS1 region from HapMapII (captured 92\% [137/149 SNPs] with $r^{2}>0.8$ and mean $\left.r^{2}=0.975\right)$.

We genotyped the three tSNPs and performed association analysis in 1985 case subjects with type 2 diabetes, 2,047 population control subjects without type 2 diabetes and 521 families. The clinical characteristics of these subjects are presented in Table 1 . There were no significant differences $(p>0.05)$ in the genotype or allele frequencies for the tSNPs between the three groups that made up the case subjects and the two groups that made up the controls (the analysis is shown in ESM Table 2). Therefore, the case and control groups were combined for analysis. There were no significant differences $(p>0.05)$ in the genotype or allele frequencies between the case and control groups for any of the tSNPs (Table 2). Table 3 shows the results of the family-based analysis using the TDT/sibTDT method [9]. There was no significant overtransmission of the minor alleles for the tSNPs in 521 families.

\section{Discussion}

This is the first large population-based case-control and family-based association study investigating common variation in the ALMS1 gene and type 2 diabetes. The HapMapII data show very good correlation between most of the SNPs across ALMS1 and this extends approximately $77 \mathrm{~kb}$ from the $5^{\prime}$ end and $11 \mathrm{~kb}$ from the $3^{\prime}$ end of the gene. Our three tSNPs captured $92 \%$ (mean $r^{2}=0.975$ ) of the common variation in the ALMS1 region from the HapMapII data. However, we found no evidence of association of variation in ALMS1 with type 2 diabetes in the case-control and family-based studies.

Our results confirm the findings of a previous small case-control study looking for association between ALMS1 and type 2 diabetes [10]. This group studied the gene variants D2672H, R2826S and R4029K in 188 type 2 diabetes patients and 167 age-matched normoglycaemic controls. Genotype and allele frequencies did not differ between patients and control subjects for gene variants $(p>0.2)$. However, this study had less power and did not involve a full SNP analysis in type 2 diabetes patients across the ALMS1 gene. Our final analysis included over 4,000 case-control subjects and 521 families and therefore had substantial power to detect odds ratios of $1.22-1.36$ that are comparable to proven type 2 diabetes susceptibility genes, such as PPARG.

$A L M S 1$ is widely expressed in tissues, including the heart and pancreas, and has been localised to centrosomes and the base of cilia [11]. The function of ALMS1 is not known, but it is thought to be involved in microtubule organisation and intracellular transport. This may have implications for understanding mechanisms of insulin secretion and the development of diabetes in the Alström syndrome. However, we have found no evidence of association between common variations of the ALMS1 gene and type 2 diabetes in the general population.

Acknowledgements This study was undertaken with the generous support of Alström Syndrome UK, the Birmingham Children's Hospital Research Foundation and Diabetes UK. We thank the International HapMap Consortium and the CEPH participants who were involved in producing the publicly available HapMap project data.

Duality of interest There is no duality of interest.

\section{References}

1. Weedon MN, Owen KR, Shields B et al (2004) Common variants of the hepatocyte nuclear factor-4alpha $\mathrm{P} 2$ promoter are associated with type 2 diabetes in the U.K. population. Diabetes 53:3002-3006

2. Alstrom CH, Hallgren B, Nilsson LB, Asander H (1959) Retinal degeneration combined with obesity, diabetes mellitus and neurogenous deafness: a specific syndrome (not hitherto described) distinct from the Laurence-Moon-Bardet-Biedl syndrome: a clinical, endocrinological and genetic examination based on a large pedigree. Acta Psychiatr Neurol Scand 34:1-35 
3. Marshall JD, Bronson RT, Collin GB et al (2005) New Alstrom syndrome phenotypes based on the evaluation of 182 cases. Arch Intern Med 165:675-683

4. Demenais F, Kanninen T, Lindgren CM et al (2003) A metaanalysis of four European genome screens (GIFT Consortium) shows evidence for a novel region on chromosome $17 \mathrm{p} 11.2-$ q22 linked to type 2 diabetes. Hum Mol Genet 12:1865-1873

5. Hearn T, Renforth GL, Spalluto C et al (2002) Mutation of ALMS1, a large gene with a tandem repeat encoding 47 amino acids, causes Alstrom syndrome. Nat Genet 31:79-83

6. Collin GB, Marshall JD, Ikeda A et al (2002) Mutations in ALMS1 cause obesity, type 2 diabetes and neurosensory degeneration in Alstrom syndrome. Nat Genet 31:74-78

7. Wiltshire S, Hattersley AT, Hitman GA et al (2001) A genomewide scan for loci predisposing to type 2 diabetes in a U.K. population (the Diabetes UK Warren 2 Repository): analysis of 573 pedigrees provides independent replication of a susceptibility locus on chromosome 1q. Am J Hum Genet 69:553-569
8. Frayling TM, Walker M, McCarthy MI et al (1999) Parentoffspring trios: a resource to facilitate the identification of type 2 diabetes genes. Diabetes 48:2475-2479

9. Spielman RS, Ewens WJ (1998) A sibship test for linkage in the presence of association: the sib transmission/disequilibrium test. Am J Hum Genet 62:450-458

10. 't Hart LM, Maassen JA, Dekker JM, Heine RJ (2003) Lack of association between gene variants in the ALMS1 gene and Type 2 diabetes mellitus. Diabetologia 46:1023-1024

11. Hearn T, Spalluto C, Phillips VJ et al (2005) Subcellular localization of ALMS1 supports involvement of centrosome and basal body dysfunction in the pathogenesis of obesity, insulin resistance, and type 2 diabetes. Diabetes 54:1581-1587 